\title{
Optimal workflow scheduling in cloud computing based on hybrid bacterial evolutionary and bees mating optimization algorithm
}

\author{
. Dinesh Kumar ${ }^{a}$, Dr Sunil Kumar ${ }^{b}$ \\ ${ }^{a}$ Research Scholar Sangam University, Bhilwara(Rajesthan) \\ ${ }^{\mathrm{b}}$ Associate Professor School of Computer Science Sangam University, Bhilwara, Rajasthan. \\ adk75mnr@gmail.com, sunil.kumar@sangamuniversity.ac.in
}

Article History: Received: 10 November 2020; Revised 12 January 2021 Accepted: 27 January 2021; Published online: 5 April 2021

\begin{abstract}
Distributed computing is the most recent developing pattern in disseminated processing that conveys equipment framework and programming applications as administrations. The clients can devour these administrations dependent on a SLA which characterizes their required QoS parameters. By using the cloud computing technique it is possible to reduce the investment on various resources like computer hardware and software. The application or processes that are hosted and executed using clouds consist of set of tasks and it is considered that this task will form the workflow. Therefore scheduling the task is considered as a major issue as resource usage has to be maximized without affecting the services that are facilitated by the cloud. In order to execute different virtual machine application of the tasks are assigned and it is termed as enterprise arranging. In the scheduling process the inter-dependent tasks are mapped and managed in the distributed resources. For additional improvement, this paper proposes a hybrid optimization algorithm for workflow scheduling (HOWS) in cloud environment. In the proposed algorithm the first contribution is the bees mating optimization (BMO) algorithm used to share physical infrastructure to enable multiple service providers to optimize scheduling. The second contribution in the proposed algorithm is the bacterial evolutionary algorithm used to flexible access of the resources in order to optimize the network resources. By combining the hybrid optimization algorithm provides the better improvement in terms of task scheduling and optimal resource allocation. The result and performance analysis shows that the proposed technique performs very efficient in terms of energy efficiency and scalability without compromising security. The performance is obtained using cloudSim tool
\end{abstract}

\section{Introduction}

Clouds are considered as huge area which consists of usable and open assets, for example, equipment, programming and application [1]. All the resources as services are delivered over internet to the client using cloud computing. The services are provided to the customers through virtualization of hardware and software [2]. By dynamically reconfiguring the resources by adjusting the variable user the load allows optimization of the resources [3].In such models the client has rights to use internet services. The users have to pay for the everyday usage. It is considered as pay-as-you-go, basis like ordinary value. Based on customized Service Level Agreements the services are facilitated in the cloud platform. Service Level Agreements will clarify the client's required Quality of Service parameters [4].Cloud figuring diminishes speculation on various assets like programming, equipment and licenses assets to be rented and discharged additionally diminishes and working and support costs.

The distributed computing is a model for empowering helpful, omnipresent and on-request arrange access so as to share pool of configurable processing assets like servers, applications, stockpiling, and systems. It is additionally utilized for help benefits that can be immediately provisioned and discharged with immaterial administration exertion. The other five characteristics of the cloud are [5]:

On-request self-administration: the customer can singularly arrangement processing abilities like network stockpiling and server time, as required consequently without human connection with specialist organizations'. Wide system get to: facilitiesexistin the network and it is able to accessthrough standard process that promotes usage by heterogeneous client platforms. Resource pooling: Distributorassets are pooled with various customers utilizing a multi-inhabitant model. As indicated by buyer request distinctive physical and virtual assets are progressively allotted and reassigned. Fast flexibility: the offices can be rapidly and flexibly provisioned in different circumstances consequently so as to scale out and quickly discharged for scale in. Elasticity is considered as the key differentiable feature which split modern clouds from other earlier utility computing forms. Measured service: it is able to command and modify the scheme automatically by using leverage meter, it has ability at some point of inattention. The services provided are storage, processing, bandwidth, and active user accounts. 
Workflow Scheduling: in cloud computing the application that are hosted and executed in its platforms composed of set of task. These tasks are used to form he workflow. The workflow process depends on the task that is performedconstructedwith the regulatorwithfactsneeds[6]. The workflow applications [7] has designed by Directed Acyclic Graph (DAG). The DAG is portrayed utilizing the tuple $G(T, E)$, where the assignment is represented by $\mathrm{T}$ and set of $\mathrm{n}$ errands can be represented as $\{\mathrm{t} 1, \mathrm{t} 2, \ldots \ldots, \mathrm{t} \mathrm{n}\}, \mathrm{E}$ demonstrate a lot of edges and it show the conditions. The ti of $\mathrm{T}$ show the assignment in the application and the edge (ti .tj) of E speaks to a

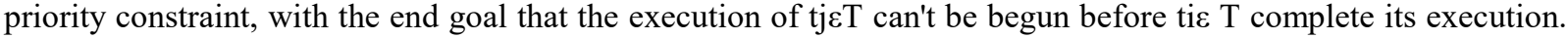
In the event that $(\mathrm{ti}, \mathrm{tj}) \varepsilon \mathrm{T}$, at that point $\mathrm{ti}$ is considered as the client of $\mathrm{tj}$ and $\mathrm{t} j$ is the offspring of ti. the figure 1 demonstrates the work flow in a cloud computing platform.

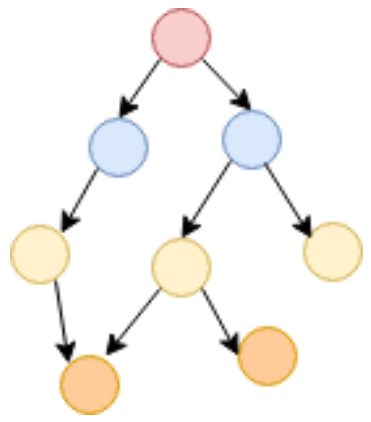

Figure 1Workflow Application

Form the figure it is clear that the tasks are inter-dependent. The first task considers as the entry task and for it there is no parents.Also, the last level of task is the exit tasks and it doesn't have children. The first task is the parent task for the level 1 and 2. Assignment of the application tasks to various VMs for execution (Wu et al 2013) is considered as the progress assigning. In this assigned process the inter-dependent progress are mapped and managed. The workflow will allocate proper resources for completing the execution process. The execution will be completed by satisfying the SLAs agreed with the users.

Workflow scheduling is a commonly used for[8] NP-broaddelinquent and numerousexperiential and metaheuristics approachesare used in the grids base system. After completing workflow task atthat its paternalerrands have finished whichafter be performed. To the available resources the workflow scheduling can distribute tasks. When the resources are available thetask are executed. For placing the task in the next resources the scheduling algorithms are used. This is commonly used in data intensive workflows in which scheduling child task process on the same resources as their parent's decreases data transfer process dramatically. During the execution process a schedule that was valid in the beginning may turn into invalid, because the resource goes offline or improve network congestion\latencies.

QoS is the aggregate exertion of administrations execution, which decides the level of the fulfillment of a client for the administrations and can be communicated as far as subjective estimates like make range (finishing time), cost, accessibility, unwavering quality, need, load parity and due date [9]. [10] have proposed a CTC calculation to accomplish lower cost while complying with the client assigned time constraint. Be that as it may, this was found to expand the general occupation fulfillment time. FIFO booking was proposed by assessing the whole gathering of undertakings in the activity line [11]. It, be that as it may, neglected to upgrade the booking to adjust to evolving loads. A heuristic put together planning with respect to PSO [12] lead to minimization of all out expense of execution yet did not take into account advancing the culmination time.

A need-based employment planning [13] model was proposed for appointing need to work process undertakings and make length, anyway we not consider the amount. In Ant Colony Optimization, a single VM which has greater implementation was used for executing all errands. This trigger the freight cumbersomeness as there would overpower trouble on one VM rest of them will be inert. The computation was seen to be not adaptable and the make range of the assignments was also broad. The PCM calculation [6] was proposed to execute the work processes with least time inside the client determined due date, yet does not address the errand consummation due date imperative. The Bi-Objective Priority based Particle Swarm Optimization [14] workflow were desined to limit execution cost while complying with a client characterized time constraint. Nonetheless, reduction of fruition duration was not examine. Meta heuristic methods designed above, it contemplate just like couple of QoS parameter [15]. Be that as it may, SLAs need various QoS indicator to met and effective work process technique are required to meet these prerequisites. Consequently, work process planning remains a key test for cloud computing models. The main objectives of the concept is summarized as follows:

- $\quad$ To develop an efficient workflow scheduling to optimizes resource utilization in Cloud computing.

- To schedule workflows on basis of multiple QoS constraints: completion time, cost and deadline 
- To achieve task load balance among the multiple VMs available in the cloud. Progress duration can be describe as duration need to complete a particular errand. VM. Completion time is the duration taken to complete the set of tasks that comprise the job or application. Cut-off point is defined as the extreme duration that can be taken to complete the application tasks. Load balancing looks at dividing the task evenly on different machine for optimum provisioning of cloud resources.

The major contributions are,

- The first contribution is to introduce a bees mating optimization (BMO) algorithm to share physical infrastructure to enable multiple service providers to optimize scheduling.

- The flexible access requires efficient bacterial evolutionary algorithm to optimize the network resources; the combined hybrid optimization algorithm provides the better enhancement in terms of task scheduling and optimal resource allocation.

\section{Related work}

Gawaliet al. [16] experientialmethodwhichmerges the MAHP, BATS + BAR optimization, LEPT, and divideand-conquer model is design to do errand assignment and device allocation. Commission arrangement and supply apportionment are major two factors of the cloud computing. In MAHP execution the errand is processed separately, by previous genuine distribution to internet capitals. By combiningBATS and BAR optimization method is used in MAHP process, the bandwidth and freight of distributor resources are major constraints.

According to Moonet al. [17] the planning calculation straightforwardly influences the expense for clients as the distributed computing gives figuring assets on a compensation for every utilization premise. Along these lines, task booking calculation dependent on insect state enhancement is presented here. In that the assignments of cloud clients are allotted for implied devices in distributed determine circumstance in an appropriate way. For improving the execution assignment to organize, present in the distributor conditions broadening and fortification procedures is utilized in the subterranean insect settlement streamlining. In underground bug state streamlining computation, the overall improvement issue with slave ants by clearing long ways whose pheromones are wrongly gathered by driving the ants.

Gonzalezet al. [18] workflows are considered as an approach to formalize and structure information examination, this will assist researchers with handling the mind-boggling activities. The utility registering is the significant qualities of cloud and this element is utilized for computational and capacity assets as a metered administration. Multitenancy is another component of distributed computing and this will empower asset and cost offering to a major pool of clients. In current information escalated examine and logical undertakings multitenancy and versatility are the significant variables. One of the key empowering agents of this combination of distributed computing and logical work processes is asset the executives. In any case, a few issues identified with information concentrated burdens, complex frameworks, for example, cross breed and multi-cloud situations to help extensive scale execution of work processes, execution variances, and dependability, present as difficulties to genuinely position mists as suitable elite foundations for logical registering. This paper shows a study on cloud asset the board that gives a broad investigation of the field. A scientific classification is proposed to investigate the chose works and the examination at last prompts the meaning of holes and future difficulties to be tended to by innovative work.

Sahniet al. [19] Cloud Computing, an appropriated registering worldview, empowers conveyance of IT assets over the cloud and pursues a compensation as-you-go charging method. Work process booking is a standout amid by testing a issues in distributor figuring. Despite the fact that, work process planning on appropriated frameworks like Grids and Clusters have been broadly examined, be that as it may, these arrangements are not feasible for a Cloud domain. It is on the grounds that, a Cloud domain varies from other appropriated condition has twice noteworthy methods: on-request asset reservation and pay-as-you-go estimating method. Accordingly, we accomplish a genuine advantages using work process organization onto internet assets paperback methodologies it may underwrite a focal points and point out a provokes explicit to a internet situation should be generated. This focused on implemented technique of dynamic financial savvy due date obliged heuristic calculation for booking a logical work process in an open Cloud. The proposed strategy plans to misuse the points of interest offered by Cloud figuring while at the same time considering the virtual machine execution fluctuation and case obtaining deferral to recognize a without a moment to spare timetable of a due date compelled logical work process at lesser expenses.

Rimal et al. [20] Multi-inhabitance can one of the significant features of dispersed registering, which gives flexibility and money related points of interest to the end-customers and pro communities by having a comparative cloud stage and its key structure with the separation of shared framework and procedure resources. In any case, resource the administrators with respect to multi-inhabitant conveyed processing is getting the chance to be a champion among the most confounding assignments as a result of the intrinsic heterogeneity and resource 
isolation. This paper proposes a novel CWSA arrangement for register concentrated work process applications in multi-inhabitant distributed computing conditions, which limits the general work process finishing time, lateness, amount of performance of a work processes, when usage of inactive internet viably. The implemented formula is focused on generating best calculations in class, i.e., FCFS, EASY Backfilling, and MCT booking arrangements to evaluate the implementation. When proof-of-idea is investigated from veritable thinking workflow models is implemented to provide adaptability of the CWSA, this checks a viability of the improved assignment. The reenactment outcome demonstrate that the implemented planning arrangement develop a work process execution and beats the previously mentioned elective booking strategies under run of the mill sending situations.

Wen et al.[21] The critical development in distributed computing has prompted expanding a distributors in internet, every one provides a administration with different terms - single term may be developed to secure a most affordable or progressively dependable. In the meantime, client applications have turned out to be increasingly intricate. Frequently, they comprise of an assorted accumulation of programming parts, and need to deal with variable outstanding burdens, which present diverse prerequisites on the framework. In this way, numerous associations are thinking about utilizing a blend of various mists to fulfill these necessities. It raises, regardless, a non-minor issue of how to pick the best blend of fogs to meet the application necessities. This paper shows a novel count to send work process applications on brought together fogs. To begin with, we familiarize an entropybased methodology with assess the most strong work process associations. Second, apply an expansion of the Bell-LaPadula Multi-Level security model to address application security necessities. At last, to enhance organization as far as its entropy and furthermore its financial cost, considering the expense of figuring power, information stockpiling and between cloud correspondence. It is actualized on the new methodology and thought about it against two existing booking calculations: EDCA and EBDLS. The calculation can discover organizations that are of equal unwavering quality however are more affordable and meet security prerequisites.

Arabnejad et al.[22] Essential science is ending up perpetually computationally concentrated, expanding the requirement for vast scale register and capacity assets, will be inside a High-Completion Computer group, and all late inside the distributor. As a rule, extensive scale logical calculation is spoken to as a work process for planning and runtime provisioning. Such booking become a considerably all the more testing issue on cloud frameworks because of the dynamic idea in the distributor, specifically, a flexibility of valuing methods (both constant and vital), the miscellaneous asset types, a immense range of administrations, and essential. This mapping of work process endeavors on to a great deal of provisioned events is an instance of the general booking issue and is NPwrapped up. Moreover, we furthermore need to ensure that certain runtime goals are met - the most regular being the cost of the figuring and the time which that computation requires to wrap up. This paper, provides a another heuristic booking calculation, BDAS, that addresses eScience work process planning under spending plan and due date limitations by Infrastructure as a Service (IaaS) mists. The interest of our work is satisfying both spending plan and due date impediments while showing a tunable cost-time trade off over heterogeneous cases. Moreover, we consider the security and intensity of our estimation by performing affectability examination. The results demonstrate that general BDAS finds a down to earth schedule for more than 40000 tests accomplishing both portrayed necessities: spending plan and due date. Moreover, our figuring achieves a 17.0-23.8 percent higher accomplishment rate when appeared differently in relation to front line estimations.

Chen et al. [23] With the wide association of disseminated figuring in various business attempts similarly as science and building spaces, shocking security organizations are continuously fundamental for taking care of work process applications with fragile transitional data. Heartbreakingly, most existing worklfow booking approaches reject the security essentials of the moderate data conveyed by work forms, and disregard the execution impact of encryption time of widely appealing data on the start of ensuing work process errands. Additionally, the idle calendar openings on resources, coming about due to data conditions among work process errands, have not been sufficient abused to direct the impact of data encryption time on work procedures' makes pans and cash related cost. To address these issues, this paper presents a novel endeavor booking framework for security fragile work forms with three novel features. In any case, we give expansive speculative examinations on how explicitly duplicating an errand's precursor assignments is helpful for staying away from both the data transmission time and encryption time from deferring undertaking's start time. By then, we portray work process errands' latest fulfillment time, and show that endeavors can be done before assignments' latest culmination time by using most economical resources for decrease cash related cost without deferring assignments' successors start time and work procedures' make ranges. In perspective on these examinations, we devise a novel arranging approach with explicit assignments duplication, named SOLID, combining two huge stages: 1) task booking with explicitly replicating herald errands to sit plan opportunities on resources; and 2) transitional data scrambling by effectively mishandling endeavors' laxity time. We evaluate our answer approach through careful execution appraisal consider using both indiscriminately made work procedures and some genuine work process pursues. Our results exhibit that the proposed SOLID approach beats existing estimations with respect to makespan, fiscal costs and resource adequacy. 
Li et al. [24]Distributed computing is a reasonable stage to execute the due date compelled logical work processes which are run of the mill huge information applications and regularly require numerous hours to wrap up. Besides, the issue of vitality utilization has turned out to be one of the significant worries in mists. In this paper, a CEAS calculation for internet organizer to minimize a processing amount of workflow and lessen a vitality utilization when fulfilling a time inhibition imperative. The CEAS calculation comprises a different branch-calculations. To begin with, the VM determination calculation it may apply the idea of amount provide to delineate to their ideal VM types by the branch-make range limitation. At that point, two undertakings consolidating strategies are utilized to diminish execution cost and vitality utilization of work process. Further, in order to reuse the dormant VM precedents which have been leased, the VM reuse technique is in like manner proposed. Finally, the arrangement of slack time recuperation is utilized to save essentialness of leased VM events. According to the time eccentrics examination, we gather that the time multifaceted nature of each subestimation is polynomial. The CEAS estimation is evaluated using Cloudsim and four genuine sensible work process applications, which shows that it outmaneuvers the related comprehended the philosophies.

Li et al. [25] Assigning administration limits in distributed computing depends on the suspicion that they are boundless and can be utilized whenever. Be that as it may, accessible administration limits change with outstanding task at hand and can't fulfill clients' solicitations whenever from the cloud supplier's point of view since cloud administrations can be shared by numerous assignments. Cloud specialist co-ops give accessible schedule vacancies to new client's solicitations dependent on accessible limits. In this paper, we consider work process planning with due date and schedule vacancy accessibility in distributed evaluate. An repeat heuristic system were exhibited for the problem under investigation which for the most part comprises of introductory arrangement development, improvement, and bother. Three beginning arrangement development techniques, two insatiable and reasonable based development procedures and bother method are proposed. Diverse procedures in the three various stage result shows in a few heuristics.

\section{Problem definition and System model}

\subsection{Problem definition}

Cloud computing is the dynamic group of assorted resources including retention, packing, webrange, computedcontrol and requestexpansionthing. These resources provide scientific, engineering and business applications services to its customers. Cloud datacenters are rich in versatile computing resources satisfying the needs of both cloud service provider and service consumer. Furthermore, cloud benefits additionally include powerfully provisioning the sharable assets among the client demand. The assets are distributed and deapportioned ideally according to the application request while thinking about asset accessibility and execution prerequisites dependent on Quality of Service parameters like vitality use, cost, time, asset use and throughput. Workflow scheduling problem is a very dynamic and random in nature. They lack prior knowledge about randomness due to unpredictable workloads, and hence, execution time and cost factors which makes this problem to be in the class of NP-hard problem, being intractable in polynomial time. Various heuristic and metaheuristic methods are used to get an optimal schedule with polynomial time complexity. The workflow scheduling plays major role in cloud computing, but fluctuating demand and dynamic nature of resources are affect the performances are efficiency and scalability. The contributions are:

- $\quad$ Bees Mating Optimization (BMO) algorithm to share physical infrastructure to enable multiple service providers to optimize scheduling.

- Bacterial Evolutionary Algorithm for flexible access of network resources

\subsection{The System Model}

The figure 2 demonstrates the framework model. IaaS cloud system were used for system model. Different information hubs include storing and calculating capitals which are present in VMs. For proper scheduling HOWS algorithm is used. Different tasksare there in the resources which are scheduled depending up on the priority. The bees mating optimization (BMO) algorithm used to share physical infrastructure to enable multiple service providers to optimize scheduling. The bacterial evolutionary algorithm used to flexible access of the resources in order to optimize the network resources. 


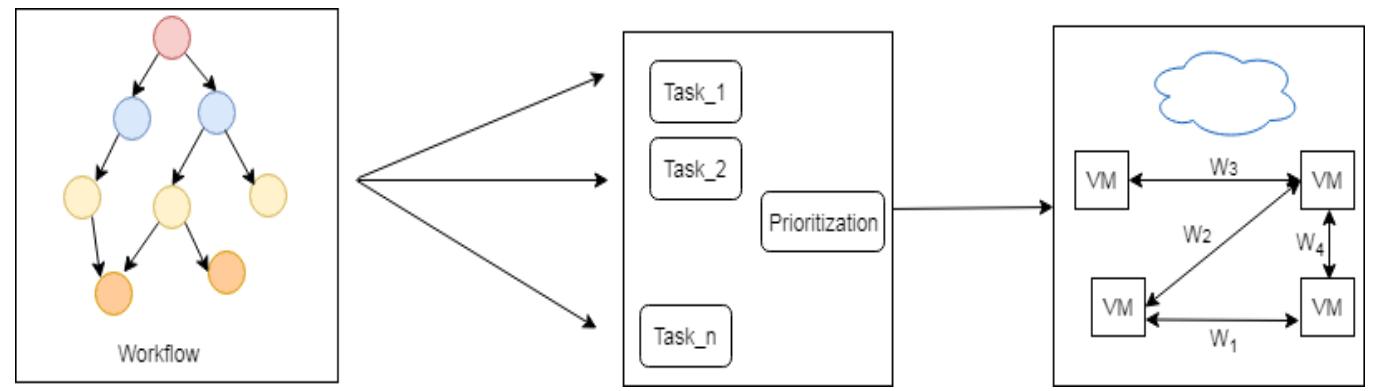

Figure 2 System Model

\section{Proposed Methodology}

Thissection describes the proposed algorithm for scheduling the workflow. It also explains the two contributions of the proposed algorithm like bee mating algorithm and evaluation bacterial algorithm.

\subsection{Bees mating optimization (BMO) algorithm}

Parameters ofmultiple service providers for optimize scheduling as follows:

User request $\left(\mathbf{U}_{\mathbf{r}}\right)$ : set of user request which consist of 1 to some number ' $n$ ' task elements.

Task Type $\left(\mathbf{T}_{\mathrm{t}}\right)$ :typeof task can be described and it consists of 1 to $\mathrm{m}$. the $\mathrm{T}_{\mathrm{m}}$ indicatemaximum number of tasks inside the task unit.

Task dependency $\left(\mathbf{T}_{\mathrm{d}}\right)$ :dependencies of task units areindicated as $U_{\mathrm{r}}$. The data obtained from $\mathrm{U}_{\mathrm{ri}}$ is given as $T_{d_{i j}}=1$ and this data is used by $T_{d_{i j}}$. Otherwise it is considered as $T_{d_{i j}}=0$

$$
T_{d_{33}}=\left[\begin{array}{cccc} 
& U_{r 1} & U_{r 2} & U_{r 3} \\
U_{r 1} & 0 & 1 & 0 \\
U_{r 2} & 1 & 0 & 0 \\
U_{r 3} & 0 & 1 & 0
\end{array}\right]
$$

Input Data $\left(\mathbf{D}_{\text {in }}\right)$ :the participatedinformationextent of task units represented as input data.

Output Data $\left(\mathbf{D}_{\text {out }}\right)$ :the productivity information magnitude of mission element is represented as $\mathrm{D}_{\text {out }}$. For this situation the source pool is accepted as heterogeneous and the source is taken from corporeal appliance or a server or PC in remote that establish the server farm. Different configurations are assigned to same resources. The resource informationconsists of six tuples and it is given as $\mathrm{R}=\left(\mathrm{PM}, \mathrm{P}_{\mathrm{c}}, \mathrm{CP}, \mathrm{R}, \mathrm{CE}, \mathrm{Nbw}, \mathrm{Ecom}\right)$.

Physical Machines (PM):it indicates the set of physical machines present in a data center and it is indicated as $\mathrm{PM}=\left(\mathrm{PR}_{1}, \mathrm{PR}_{2}, \mathrm{PR}_{3}, \ldots, \mathrm{PR}_{\mathrm{n}}\right)$.

Computing Power $\left(\mathbf{P}_{\mathrm{c}}\right): \mathrm{P}_{\mathrm{c}}$ indicate the matrix of computing power of the PM. For executingthe errand unit type i on physical device $\mathrm{PM}_{\mathrm{j}}$ is indicated by $P_{c_{i j}}$ and the average power of $\mathrm{PM}_{\mathrm{j}}$ is indicated by $P_{c a v g, j}$. The average power is calculated by taking the mean of component in vertical matrix $P_{c_{j}}$.

$$
T_{d_{i j}}=\left[\begin{array}{ccccc} 
& P M_{1} & P M_{2} & \cdots & P M_{j} \\
U r_{1} & T_{t 1,1} & T_{t 1,2} & \cdots & T_{t 1, j} \\
U r_{2} & T_{t 2,1} & T_{t 2,2} & \cdots & T_{t 2, j} \\
\vdots & \vdots & \vdots & \vdots & \vdots \\
U r_{i} & T_{t i, 1} & T_{t i, 1} & \cdots & T_{t i, j}
\end{array}\right]
$$

RAM in PM (R):the existing RAM or storage size of separate PM.

Computing Energy(CE): A matrix which indicates theexecution time of a task unit. The energy absorb by a $\mathrm{PM}_{\mathrm{j}}$ to implement $\mathrm{i}$ task unit type per unit time per unit information is given asCEij.

Bandwidth (BW): the bandwidth between PMs and, the information transfer ratio between $\mathrm{PM}_{\mathrm{i}}$ to $\mathrm{PM}_{\mathrm{j}}$ and this indicated by $\mathrm{BW}_{\mathrm{ij}}$. 


\subsection{BMO Algorithm}

Initially it is considered that there are $\mathrm{k}$ subtasks for the users in the cloud services. This $\mathrm{k}$ subtasks are scheduled using $m$ computational resourcesand it is indicated by $\left\{R_{1}, R_{2}, \ldots, R_{m}\right\}$.

It is considered that each resource, $R_{j}$ hasfixed costsprice and it is indicated $a s p_{j}$. The price vector is given by $p$ $=\left\{\mathrm{p}_{1}, \mathrm{p}_{2}, \ldots . \mathrm{pm}\right\}$.The time allocated for $\mathrm{R}_{\mathrm{i}}$ to execute the subtask is given $\mathrm{t}_{\mathrm{i}}$. The execution time vector is given by $\mathrm{t}$ $=\left\{\mathrm{t}_{1}, \mathrm{t}_{2}, \ldots . \mathrm{t}_{\mathrm{m}}\right\}$.

To optimize the swarm-based honey bee mating algorithm is usedand the optimization is based on thelook calculation is empowered by the way toward mating in genuine bumble bees. The conduct of bumble bees is the cooperation of their::

I. Genetic probability

II. Ecological and physiological conditions

III. The social states of the province, just as different prior and continuous cooperations between these three parameters.

The arrangements of move make place by every honey bee areunfolded as per hereditary, environmental, and social states of the settlement. The five steps included in the algorithm are:

1. The computation starts with the mating- flight, where a ruler (best game plan) picks meanders probabilistically to shape the spermatheca (once-over of machines). A machine is then looked over the summary at sporadic for the development of broods.

2. Creation of new broods (fundamental game plans) by cross overring the drones ${ }^{\text {ee }}$ genotypes with the queen ${ }^{\text {ee }}$.

3. Use of workers (heuristics) to conduct local search on broods (trial solutions).

4. Adaptation of workers ${ }^{\text {ee }}$ capability is depend on cost of development attain on broods

5. Replacement of flimsier rulers by fitter broods.

Honey bees are a remarkable assortment of social creepy crawlies and they live in hives framed by them. The three regular components in the hive are one ruler, a few automatons and the laborers. Because of its encouraged illustrious jam the extent of the ruler is bigger than different honey bees. Normally ruler's life expectancy is around five to six years and it has the ability to set down 1500 eggs. While the ruler flies outside and a long way from the hive, the automaton attempts to mate with her noticeable all around. The working standard of the BMO is given the figure 2 .

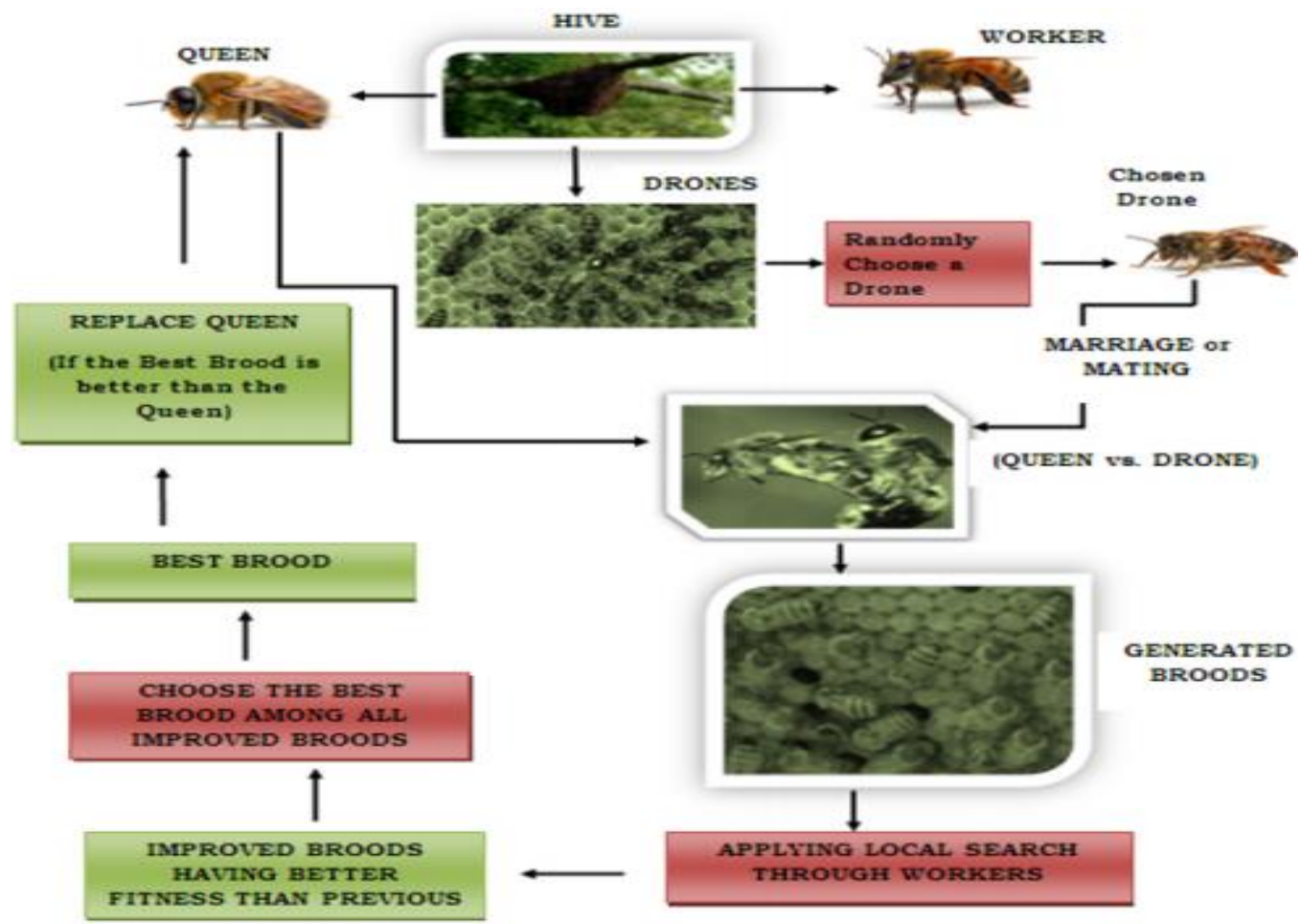

Figure 2 BMO Algorithm

The equation (3) shows the controlled mating vibration on queen. 


$$
P(D)=e^{\left[\frac{-\Delta(f)}{S(t)}\right]}
$$

where $\mathrm{P}(\mathrm{D})$ determine the possibility totaling a vibration sperm (D) to the queen's spermatheca, $\Delta(\mathrm{f})$ indicate the complete alteration among the vibration and queen's spermatheca, and queen's fitness.

\begin{tabular}{|c|c|}
\hline \multicolumn{2}{|c|}{ Algorithm 1BMO algorithm for to optimize scheduling. } \\
\hline Input & $\mathrm{D}_{\mathrm{in}}, \mathrm{Ur}, \mathrm{T}_{\mathrm{t}}, \mathrm{BW}$ \\
\hline Output & Multiple Service Providers To Optimize Scheduling \\
\hline 1 & Initialize $\mathrm{D}_{\mathrm{in}}, \mathrm{U}_{\mathrm{r}}, \mathrm{T}_{\mathrm{t}}, \mathrm{BW}$ \\
\hline 2 & Calculate the $\mathrm{U}_{\mathrm{r}}$ and $\mathrm{D}_{\text {in }}$ (using it equation) \\
\hline 3 & Calculate the $T_{d}$. \\
\hline 4 & Remove the depended data from the vector. \\
\hline 5 & Calculate the initial value of $P(D)$ \\
\hline 6 & $\operatorname{if}(P(D)==1)$ \\
\hline 7 & $\mathrm{U}_{\mathrm{r}}$ with higher priority is executed \\
\hline \multirow[t]{2}{*}{8} & Calculate the $\mathrm{P}_{\mathrm{c}}, \mathrm{CE}$ \\
\hline & Keep it best solution \\
\hline 9 & else \\
\hline 10 & Wait until P(D) become high \\
\hline 11 & After some iteration if $(P(D)==0)$ \\
\hline 12 & Replace the queen with brood \\
\hline 17 & End \\
\hline
\end{tabular}

ReturnMultiple Service Providers To Optimize Scheduling

The queen's speed at time' $t$ ' is given by $\mathrm{S}(\mathrm{t})$. It is considered that in the mating procedure the ruler's likelihood with mating speed stays bigger in the underlying stage and furthermore if the automaton's wellness is equivalent to the ruler's wellness. After cycle, the speed and vitality of the ruler decreases and the equation is given (2) and (3):

$$
\begin{aligned}
& \boldsymbol{S}(\boldsymbol{t}+\mathbf{l})=\boldsymbol{\alpha} \boldsymbol{S}(\boldsymbol{t}) \\
& E(t)=\alpha E(t)
\end{aligned}
$$

Where, $\alpha$ indicate the arbitrarilyengenderedissue and its assortmentsamong $[0,1]$ and it calculate the decreased movement and charge at single iteration. $E$ indicate the charge and ' $\mathrm{S}$ ' indicate the movement.

\subsection{Bacterial Evolutionary Algorithm}

In cloud computing the main aim is to optimal utilization of the resources. To utilize the resources the major factor is toreducemakespani.e, to boost the efficiency of the cloud systems.Another factor is the reduced cost of using the available resources.

$$
\begin{aligned}
& \operatorname{Cost}=\min \left(\mathrm{C}\left(\mathrm{r}_{\mathrm{i}}, \mathrm{j}_{\mathrm{k}}\right)\right) \text { for } 1 \leq \mathrm{i} \leq \mathrm{n}, 1 \leq \mathrm{k} \leq \mathrm{m} \\
& \operatorname{makespan}=\min \left(F_{j \mathrm{j}}\right) \text { for } j_{\mathrm{i}} \in \mathrm{J}
\end{aligned}
$$

$$
\text { Fitness Function }=\alpha \operatorname{cost}+\beta \text { makespan }+\gamma \text { reliabilty }
$$

Where, $0 \leq \alpha \leq 1,0 \leq \beta \leq 1$ and $0 \leq \gamma \leq 1$ are hefts to arrangewith themechanismsincluding the fitness job. Cost $\mathrm{C}\left(\mathrm{r}_{\mathrm{i}}, \mathrm{j}_{\mathrm{k}}\right)$ is the cost of the jobj $\mathrm{j}_{\mathrm{k}}$ which executes on resource $\mathrm{r}_{\mathrm{j}}$ and make span is the concludingperiod of the occupation. 
Thus, for the proper optimization of the resources in the network the bacterial evolutionary algorithm is used.The major steps in the bacterial evolutionary algorithm are chemo taxis, swarming, imitation, removal and scattering. The food searching behavior of the bacteria is used for forming the bacterial evolutionary algorithm. Suppose to initiate charge inhalation per duration(E/T) is maximized.

Chemo taxis:the position of the E.Coli germs changes by dip and collapse through flagella location in this process. If the direction of movement of bacterium is same for certain interval of time then it is dip. Similarly,if the movement of bacterium various for a period of time it is tumbling. The $\mathrm{p}^{\text {th }}$ bacteriumatq ${ }^{\text {th }}$ chemotactic $^{\text {th }}$ reproductive and $\mathrm{t}^{\text {th }}$ elimination and dispersal step is given by $\alpha^{\mathrm{p}}(\mathrm{q}, \mathrm{r}, \mathrm{t})$. The step size of tumble is given by $\mathrm{S}(\mathrm{p})$. The computation chemo taxis is described as:

$$
\alpha^{p}(q+1, r, t)=\alpha^{p}(q, r, t)+S(p)
$$

Swarming: set of microorganismscan be shifted to nutrientsbut then againthis can moreover be fascinated or forbidden. In particular time of stress only the bacterium can be attracted.

Reproduction: After chemo taxis process, the next process is the reproduction. The fitness value of the bacterium is calculated in this process. The fitness value is classify based on arise order. To maintain a size of the crowd as stable it reduce a wealthy germs and lead to passaway. After wealthy germs it will separate asexually into two.

Elimination and dispersal: The elimination dispersal method were used for local optima

The parameter used in the BEA algorithm are: $\mathrm{d}$ - measurement of the hunt space, $\mathrm{N}$ - number of microbes in the, $N \mathrm{c}$ - chemotaxis step, $N s$ - swim length, $N \mathrm{r} e$ - the quantity of multiplication steps, $N e$ d: the quantity of disposal dispersal occasions, Ped - end dispersal with likelihood, S (I): the span of the progression taken the arbitrary way.

\begin{tabular}{|c|c|}
\hline \multicolumn{2}{|c|}{ Algorithm 1BEAfor Network Resource Utilization } \\
\hline Input & $\mathrm{d}, \mathrm{N}, N_{d}, N_{t}, N s f, N f e, P f e, \mathrm{~S}$ \\
\hline Output & Optimal Resource utilization \\
\hline 1 & Initialization of the parameters: $\mathrm{d}, \mathrm{N}, N_{c}, N_{\mathrm{s}}, N_{\mathrm{re}}, N_{\text {ed }} P_{\text {ed }} \mathrm{S}(\mathrm{i})$ \\
\hline 2 & Elimination-dispersal loop is taken by $t=t+1$ \\
\hline 3 & Reproduction loop is represented by: $r=r+1$ \\
\hline 4 & Chemo taxis loop is given by $\mathrm{q}=\mathrm{q}+1$ \\
\hline 5 & $\begin{array}{l}\text { Select the heuristic. } \\
\text { Calculate fitness function } J(p, q, r, t) \text { and it is given by } \\
J(p, q, r, t)=J(p, q, r, t)+J_{c c}\left(\alpha^{P}(q, r, t), L(q, r, t)\right)\end{array}$ \\
\hline 6 & Assume $J_{\text {last }}=J(p, q, r, t)$ \\
\hline 7 & Tumble: generate a random vector $\Delta(\mathrm{i}) \in R^{\mathrm{n}}$ with each $\Delta_{\mathrm{m}}(\mathrm{p}), \mathrm{m}=1,2,3 \ldots . . \mathrm{d}$. \\
\hline 8 & Move: $\alpha^{P}(\mathrm{q}+1, \mathrm{r}, \mathrm{t})=\alpha^{P}(\mathrm{q}, \mathrm{r}, \mathrm{t})+\mathrm{S}(\mathrm{P})\left(\Delta(\mathrm{P}) / \mathrm{V} \Delta^{T}(\mathrm{P}) \Delta(\mathrm{P})\right)$ \\
\hline 9 & Compute $J(p, q, r, t)$ and $J(p, q, r, t)+J_{c c}\left(\propto^{P}(q, r, t), P(q, r, t)\right)$ \\
\hline 10 & $\begin{array}{l}\text { Swim } \\
\text { Assume } \mathrm{m}=0 \text {. } \\
\text { While } \mathrm{m}<N_{\mathrm{s}, \mathrm{m}}=\mathrm{m}+1 \\
\text { if } J(\mathrm{p}, \mathrm{q}+1, \mathrm{r}, \mathrm{t})<J_{\text {last }} \text { let } J_{\text {last }}=J(\mathrm{p}, \mathrm{q}+1, \mathrm{k}, \mathrm{l}) \text { and } \alpha^{P}(\mathrm{q}+1, \mathrm{r}, \mathrm{t})=\alpha^{P}(\mathrm{q}, \mathrm{r}, \mathrm{t})+\mathrm{S}(\mathrm{P})(\Delta(\mathrm{P}) / \\
\left.\mathrm{V} \Delta^{T}(\mathrm{P}) \Delta(\mathrm{P})\right) \text { and use this } \alpha^{P}(\mathrm{q}+1, \mathrm{r}, \mathrm{t}) \text { to compute the new } \mathrm{J}(\mathrm{p}, \mathrm{q}+1, \mathrm{r}, \mathrm{t})\end{array}$ \\
\hline 11 & else let $\mathbf{m}=N_{s}$ \\
\hline 12 & end \\
\hline 13 & Go to next bacteria $(p+1)$ if $p \neq N$. \\
\hline 14 & End \\
\hline
\end{tabular}

\section{Simulation Results}


Using the real world workflow the experimental analysis to evaluate the proposed algorithm is described here. The terms like makespan, security risk, and security threatare used to evaluate the performance of the proposed algorithm. The comparison is performed with the existing algorithm WOWS.

In this paper, the analysis needs to be repeatable for comparethe algorithm with various parameters. Actualizing exploratory circumstances is costly and furthermore difficult to do as asset conditions changes from time to time because of the cloud shared infrastructure. Thus choice of reenactment instruments is additionally a significant factor. The cloud recreation instrument is utilized to reproduce work process the board and it additionally plan a dynamic cloud condition.

Data Sets: in this, we have selected five various world scientific progress approach. Center.

CyberShake: in order to verify the earthquake alarms this were used by the Southern California Earthquake

Epigenomics: to generate multiple lanes of DNA sequences it is used by the DNA sequence lanes

Montage: to collect multiple input imagesof the sky to create custom mosaics. It is created by NASA/IPAC stitches.

Inspiral: reason for this informational index is to make and examine gravitational waveforms from information gathered amid the combining of conservative parallel frameworks.

Sipht [58]: it is usually utilized in bioinformatics. It is utilized to look for little untranslated bacterial administrative RNAs.

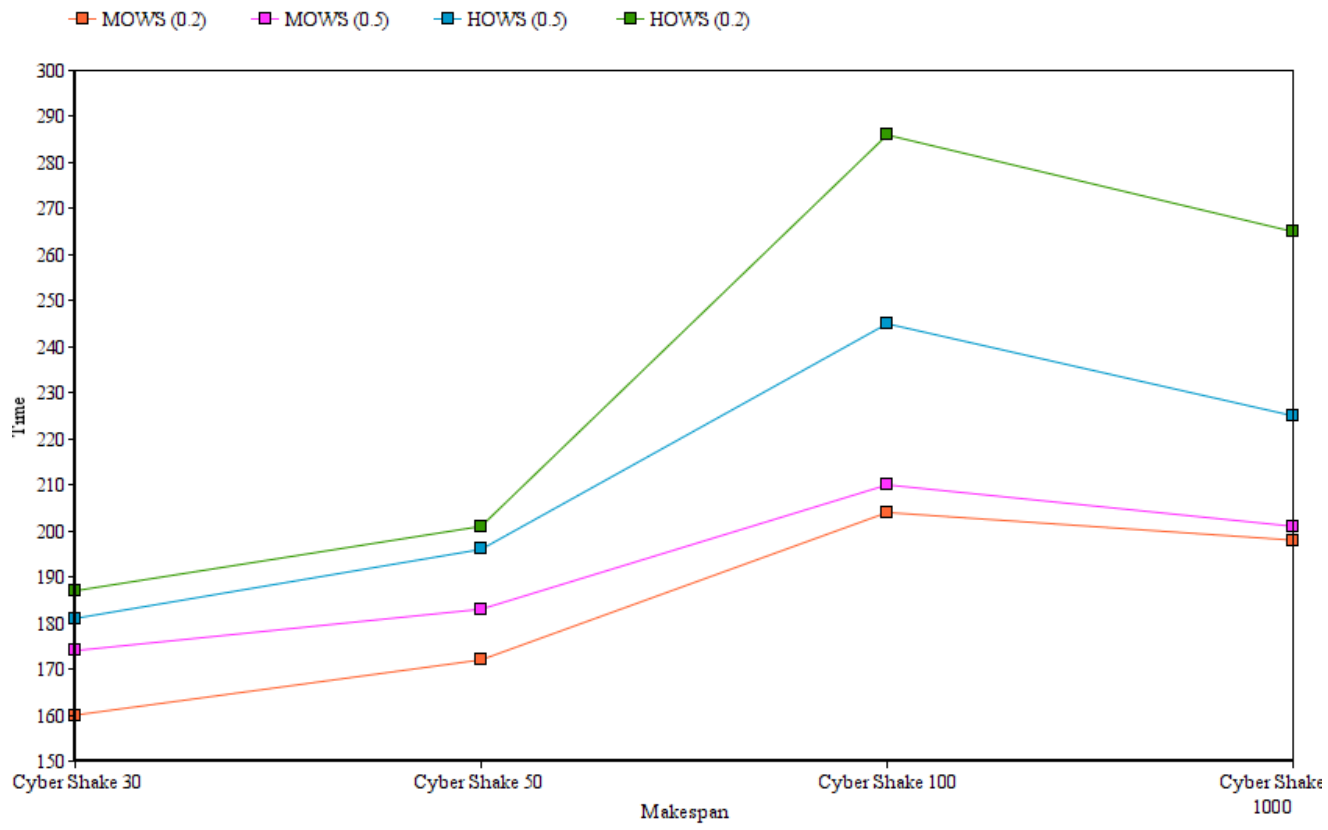

Figure 3 Makespan Analysis

The functionality of the analysis is given by taking CyberShake progress with 30, 50, 100, and 1000 errand. HOWS with $\mathrm{W}_{\mathrm{Sec}}=0.2$ andW $\mathrm{W}_{\mathrm{Sec}}=0.5$. MOWS with $\mathrm{W}_{\mathrm{Sec}}=0.2$ and $\mathrm{W}_{\mathrm{Sec}}=0.5\left(\mathrm{~W}_{\mathrm{Sec}}\right.$ identifies the effect of privacy or achievement from the user's side). HOWS and MOWS are the algorithm used for the schedulingtheworkloadson a heterogeneous distributor infrastructure with 20 material and 100virtual devices. 
$\square$ - MOWS (0.2) $\quad \square$ - MOWs (0.5) $\quad \square$ HOWs (0.5) $\quad \square$ - HOWS (0.2)

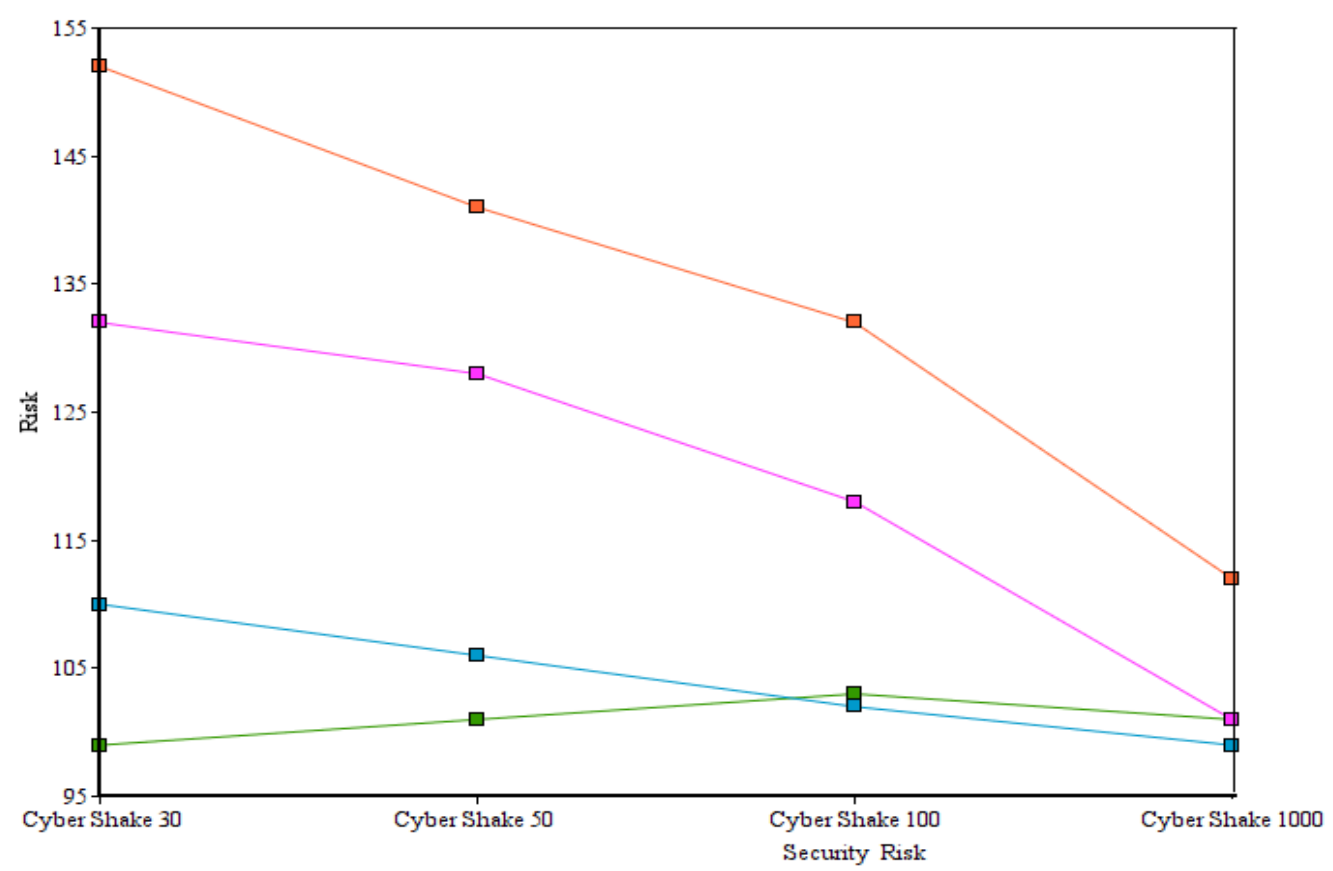

Figure 4 Security Risk Analysis

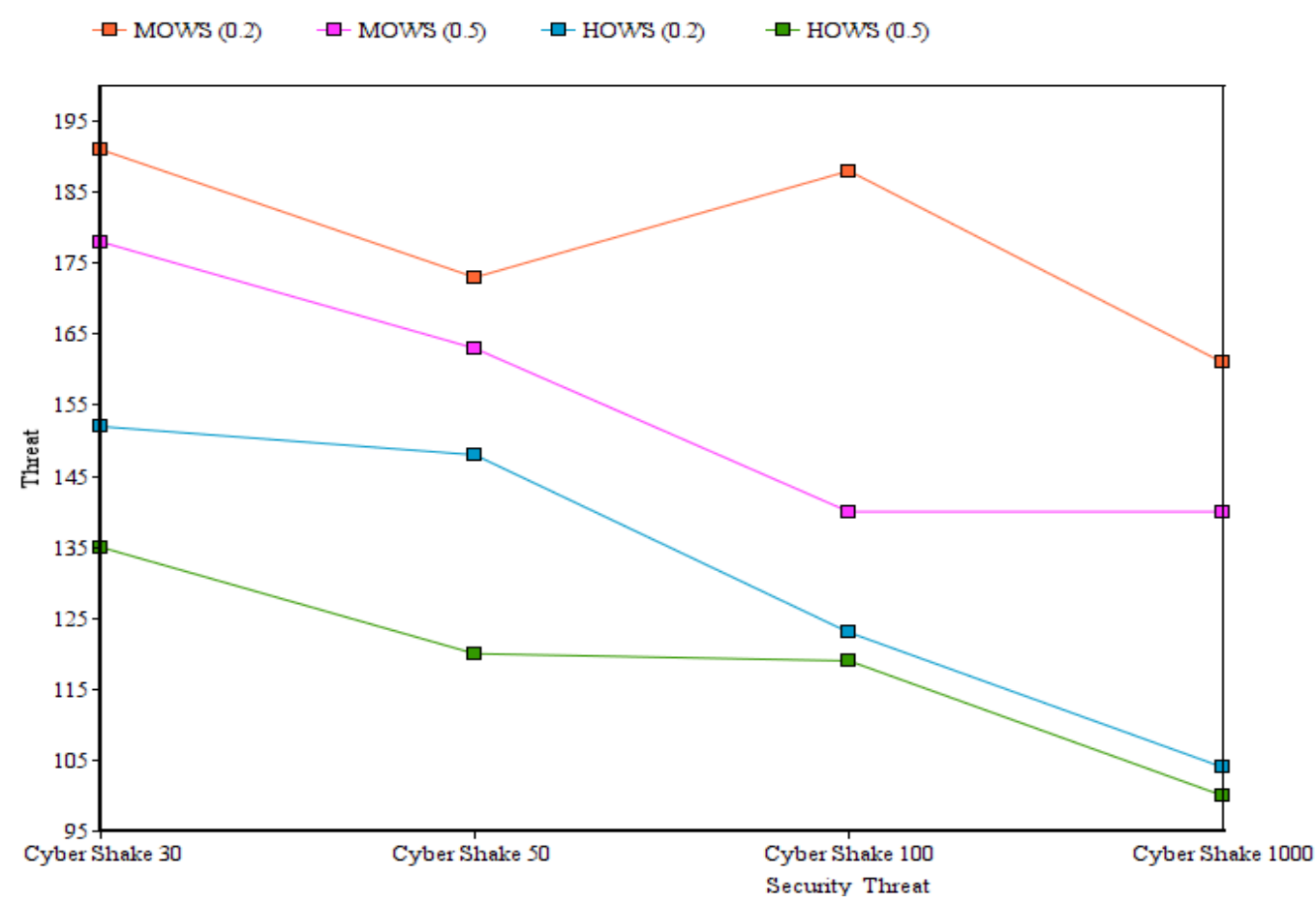

Figure 5 Security Threat Analysis 


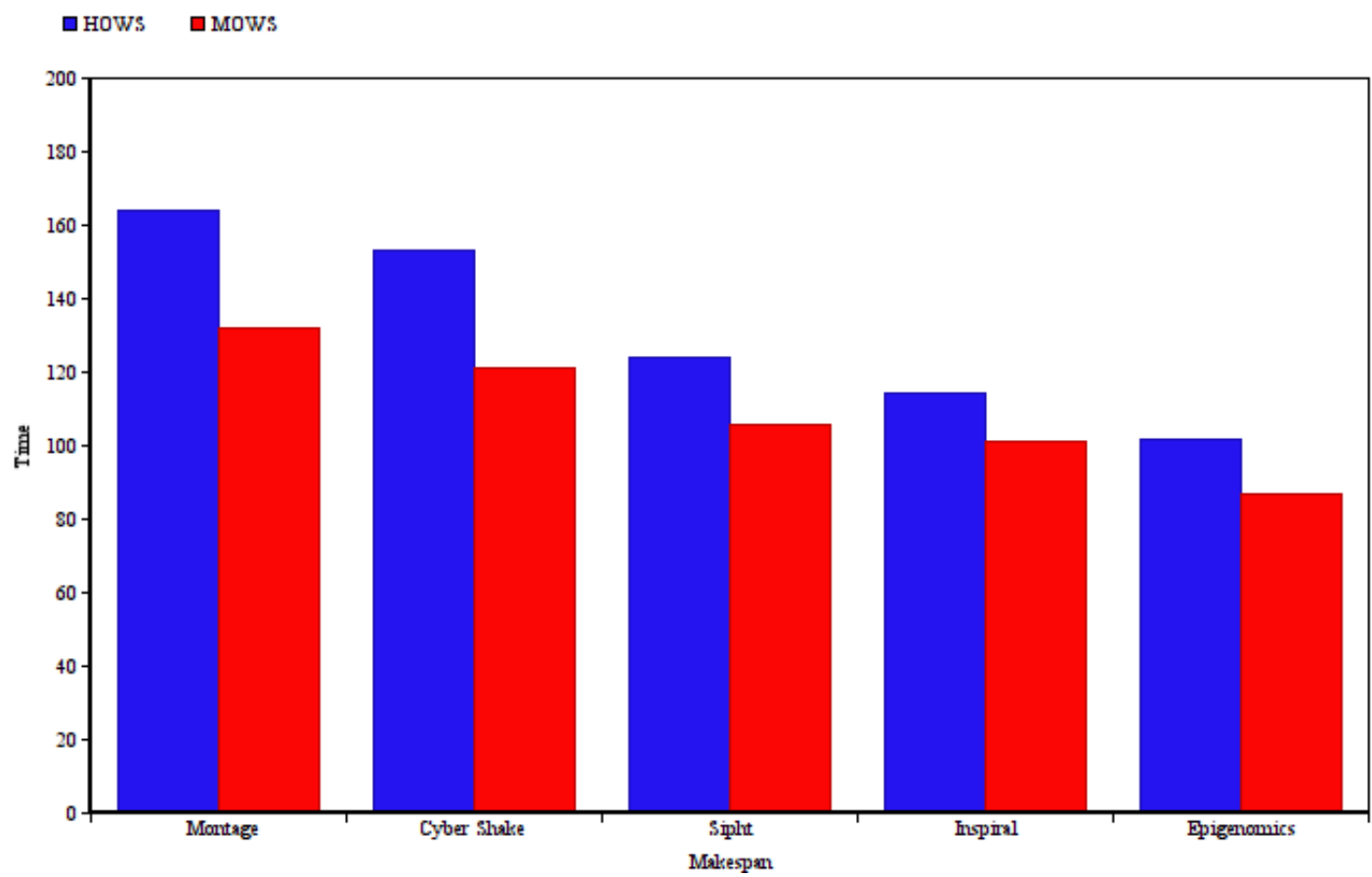

Figure 6 Comparison of Makespan

口Hows $\square$ Mows

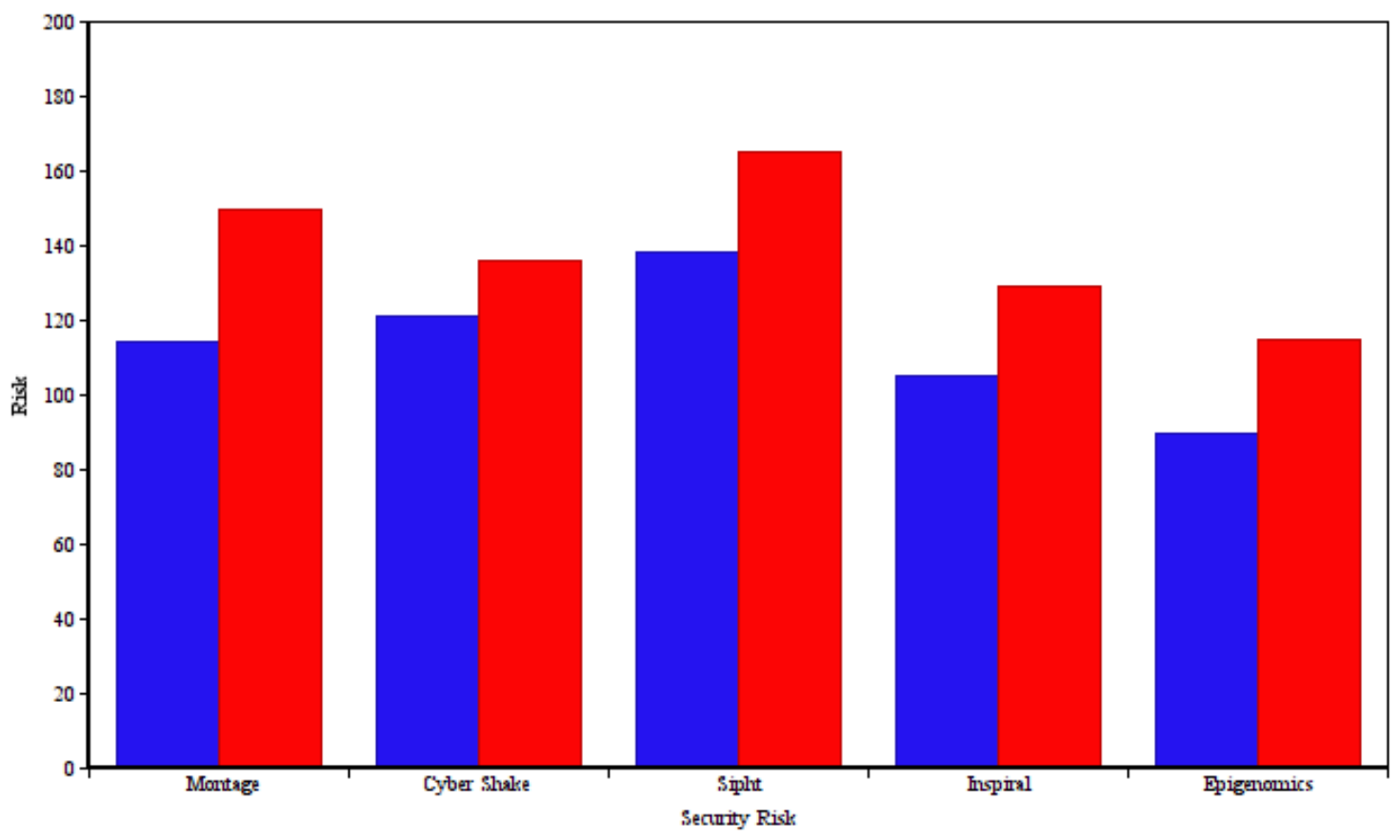

Figure 7Comparison ofSecurity Risk 


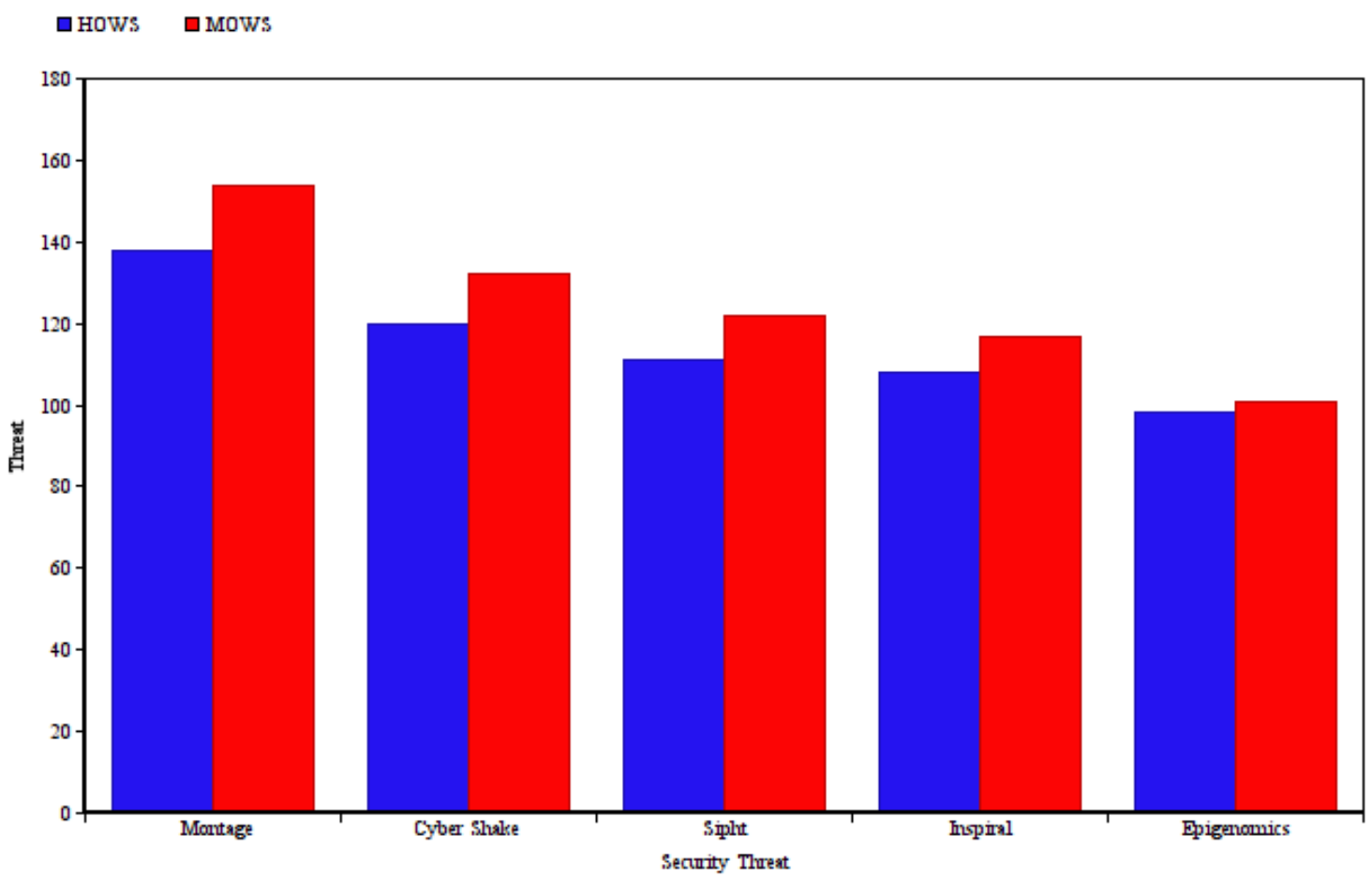

Figure 8 Comparison of Security Threat

Results the execution of the calculations is examined situated as far as makespan, security warning and security chance. Figure 3 shows that makespan of outstanding tasks at hand in the majority of the two calculations are nearly the equivalent. The examination among algorithmsbased on the security hazard is outlined in Figure 4. In HOWS the normal likelihood of CyberShake work process being assaulted amid its execution is less than110, anyway this likelihood is a lot higher in MOWS. Figure 5highlights HOWS with WSec $=0.5$ gives less security danger to all ofthe outstanding tasks at hand.

\section{Conclusion}

For cloud environment, data transferring between tasks is aninevitable. In cloud the security is considered as an important factor for scheduling. The task scheduling is an important procedure in the cloud platform. Different scheduling algorithms are used in cloud for the scheduling and it is described in the section 1 and section 2 . In this paper hybrid optimization algorithm for workflow scheduling (HOWS) algorithm is used for scheduling the task. For enhance the performances of scheduling in cloud two algorithms are used. The bees mating optimization (BMO) algorithm used to share physical infrastructure to enable multiple service providers to optimize scheduling. Also the bacterial evolutionary algorithm used to flexible access of the resources in order to optimize the network resources. By combining the hybrid optimization algorithm provides the better improvement in terms of task scheduling and optimal resource allocation. The simulation shows that the HOWS is having low makespan and low security risk and threat. Thus, from the analysis it is clear that the proposed algorithm is having better performance

\section{References}

Zhong, G \& Yuan, T 2013, 'Provisioning Virtual Educational Resources Based on Private Cloud', Proceeding of the International Conference on Information Engineering and Applications, Springer, vol. 1, no. 42, pp. 345356.

Khaled, AKM, Kirley, M \&Buyya, R 2009, ‘ Multi-Objective Differential Evolution for Scheduling Workflow Applications on Global Grids', Journal Concurrency and Computation: Practice and Experience, vol. 21, no. 13 , pp. 579-586.

Randles, M, Lamb, D \&Bendiab, A 2010, 'A Comparative Study into Distributed Load balancing Algorithms for Cloud Computing', Proceedings of IEEE 24th International Conference on Advanced Information Networking and Applications Workshops, pp. 551-556.

Tsai, T, Chen, Y, Huang, H, Huang, P \& Chou, K 2011,'A practical Chinese wall security model in cloud computing', APNOMS, pp. 478-487.

Shrivastava, N \& Yadav, R 2013, 'Cloud Security for Computing Secure Cloud Bursting, Brokerage and Aggregation Using Cryptography’, IJCSMC, vol. 2, no. 9, pp. 24 - 28. 
Abrishami, S \&Naghibzadeh, M 2012, 'Deadline-constrained workflow scheduling in software as a service Cloud', Scientia Iranica, Elsevier, vol. 19, no. 3, pp. 680-689.

Kumar, N , Sharma, P \& Krishna, V 2012, 'Load Balancing of Nodes in Cloud Using Ant Colony Optimization', Proceedings of the 14th International Conference on Modelling and Simulation, pp. 3-8.

Elzeki, OM, Rashad, MZ \&Elsoud, MA 2012, 'Overview of Scheduling Tasks in Distributed Computing Systems', International Journal of Soft Computing and Engineering, vol. 2, no. 3, pp. 143-151.

Yee, MC \& Peng, YJ 2012, 'A QoS Aware Services Mash up Model for Cloud Computing Applications', Journal of Industrial Engineering and Management, vol. 5, no. 2, pp. 457-472.

Liu, K, Jin, H, Chen, J, Liu, X, Yuan, D \& Yang, Y 2010, 'A compromised Time-Cost Scheduling Algorithm in SwinDeW-C for Instance-Intensive Cost-Constrained Workflows on Cloud Computing Platform', International Journal of High Performance Computing Applications , vol. 4, no. 2, pp. 445-456.

Yujiage\&Guiyiwei 2010, 'GA - Based Task Scheduler For the Cloud Computing Systems', Proceedings of the International Conference on Web Information Systems and Mining, vol. 02, pp. 181 - 186.

Pandey, S, Wu, L, Guru, M \&Buyya, R 2010, 'A Particle Swarm Optimization-based Heuristic for Scheduling Workflow Applications in Cloud Computing Environments', Proceeding of the IEEE International Conference on Advanced Information Networking and Applications, pp. 400-407.

Shamsollah, G \& Othman, M 2012, 'Priority based Job Scheduling Algorithm in Cloud Computing', Proceedings of the International Conference on Advances Science and Contemporary Engineering, pp. 778 - 785 .

Amandeep, V 2014, 'Bi-Criteria Priority Based Particle Swarm Optimization Workflow Scheduling Algorithm for Cloud', Proceedings of the International Conference on Recent Advances in Engineering and Computational Sciences, pp. 06-09.

Rak, M \&Venticinque, S 2009, 'QoS Management in Cloud@Home Infrastructures', Proceedings of the Simulation Modelling Practice and Theory, pp. 1378-1398.

Gawali, MahendraBhatu, and Subhash K. Shinde. "Task scheduling and resource allocation in cloud computing using a heuristic approach." Journal of Cloud Computing 7.1 (2018): 4.

Moon, YoungJu, et al. "A slave ants based ant colony optimization algorithm for task scheduling in cloud computing environments." Human-centric Computing and Information Sciences 7.1 (2017): 28.

Gonzalez, Nelson Mimura, Tereza Cristina Melo de Brito Carvalho, and Charles Christian Miers. "Cloud resource management: towards efficient execution of large-scale scientific applications and workflows on complex infrastructures." Journal of Cloud Computing 6.1 (2017): 13.

Sahni, Jyoti, and Deo Prakash Vidyarthi. "A cost-effective deadline-constrained dynamic scheduling algorithm for scientific workflows in a cloud environment." IEEE Transactions on Cloud Computing 6.1 (2018): 2-18.

Rimal, Bhaskar Prasad, and Martin Maier. "Workflow scheduling in multi-tenant cloud computing environments." IEEE Transactions on parallel and distributed systems 28.1 (2017): 290-304.

Wen, Zhenyu, et al. "Cost effective, reliable and secure workflow deployment over federated clouds." IEEE Transactions on Services Computing 10.6 (2017): 929-941.

Arabnejad, Vahid, Kris Bubendorfer, and Bryan Ng. "Budget and Deadline Aware e-Science Workflow Scheduling in Clouds." IEEE Transactions on Parallel and Distributed Systems 30.1 (2019): 29-44.

Chen, Huangke, et al. "Scheduling for workflows with security-sensitive intermediate data by selective tasks duplication in clouds." IEEE Transactions on Parallel and distributed systems 28.9 (2017): 2674-2688.

$\mathrm{Li}$, Zhongjin, et al. "Cost and energy aware scheduling algorithm for scientific workflows with deadline constraint in clouds." IEEE Transactions on Services Computing 11.4 (2018): 713-726.

Li, Xiaoping, Lihua Qian, and Rubén Ruiz. "Cloud workflow scheduling with deadlines and time slot availability." IEEE Transactions on Services Computing 11.2 (2018): 329-340. 